\title{
Application of a 3-D CFD model to investigate flood-related engineering problems
}

\author{
Daniel Horna Munoz ${ }^{1,}$ and George Constantinescu ${ }^{2}$ \\ ${ }^{1}$ Environmental Engineering Department, Universidad de Ingenieria y Tecnologia, Lima, Peru \\ ${ }^{2}$ Civil and Environmental Engineering Department, University of Iowa, Iowa, USA
}

\begin{abstract}
The paper discusses the application of a fully 3-D, nonhydrostatic, RANS model with deformable free-surface capabilities to several main types of flood-related engineering problems. The model is found to accurately simulate the dam break flow developing in a $90^{\circ}$ open channel bend for which detailed validation data from a laboratory experiment are available. First practical application deals with mitigation of flooding extent via implementation of flood protection structures such as floodwalls. Numerical results show that the floodwalls effectively protect the critical regions situated in the vicinity of the river and the reduction of the flooded area with respect to the case when floodwalls are not present is about the same at high-flow conditions and at low-flow conditions. The second application deals with prediction of flow structure around a location containing a bridge whose deck becomes submerged as the flood wave passes the bridge location. The final application considers the effects of a sudden dam break failure for a dam situated upstream of the Iowa City, USA.
\end{abstract}

\section{Introduction}

Numerical models generally employed to study flood-related problems are based on solving the 1-D or the 2-D Saint-Venant equations. For many applications, these models are able to reproduce fairly well the unsteady flood wave propagation including the free-surface elevation profiles and the depth-averaged velocity field. Still, to provide accurate predictions these models need to be calibrated. In regions where 3-D effects (e.g., where strong vertical nonuniformity of the flow and/or strong cross-stream secondary currents are present) become important, such as near hydraulic structures, near river confluences or in high curvature regions of streams, the accuracy of these models is generally not very good, as the main modelling assumptions are violated. For such cases, one should consider the use of a 3-D, non-hydrostatic model with deformable free-surface capabilities.

In the present paper we show that such a 3-D RANS model using the Volume-of-Fluid method to calculate the free surface position can be employed for several main types of applications in which a flood wave is generated. The model is built within an existing commercial software (STARCCM+).

\footnotetext{
Corresponding author: dhorna@utec.edu.pe
} 
The parallel code is highly scalable and can be run efficiently for applications involving tens of millions of grid points. Horna-Munoz and Constantinescu (2016) provide a description of the numerical model, main type of boundary conditions and gridding techniques. In the present paper, we exemplify the use of

the solver for several other types of applications that are relevant for flood related problems.

The first case corresponds to a dam break wave propagating in a $90^{\circ}$ bend that was previously simulated using 2-D and 3-D models and for which detailed validation data are available. The second case exemplifies how the numerical model can be used for flood mitigation in an urban riverine environment. Flood protection walls were included in an area of high economic development that needs to be protected against flooding. The third test case used the 3-D model to investigate pressure scour effects associated with flood propagation that can induce temporary submergence of a bridge deck. The last test case exemplifies the use of the 3-D model to predict a realistic dam break event for the Coralville Dam, which is a flood-protection dam located upstream of Iowa City, USA.

\section{Results}

Horna-Munoz and Constantinescu (2016) discuss several validation test cases for steady and unsteady flow in channels and domains containing natural river reaches in which an unsteady hydrograph is imposed at the upstream boundary. Given that another main type of applications intended for this model is prediction of dam break flows in natural environments, validation for this type of flows is required. The chosen test case is a dam break flow generated at the upstream end of a $90^{\circ}$ bend that was studied experimentally by SoarezFrazao and Zech (2002). Figure 1 shows the general layout of the experiment and some of the main geometrical parameters. Figure 2 compares the numerical predictions of the free surface position at three relevant times during the propagation of the flood wave. The present 3-D simulation results conducted on a mesh containing about 6 million cells are in good agreement with the experimental data and their level of accuracy is comparable to that of a 3-D simulation performed by Biscarini et al. (2010) using a different model. Both 3-D models were run with the same spatial and temporal resolution. Cell sizes had edge size of $0.01 \mathrm{~m}$ with mesh refinement of 30 wall units near solid surfaces and a time step of $0.02 \mathrm{~s}$. The proposed 3-D model was run with 64 processors and the solution took 3 days approximately. Though not included, simulations performed using a 2-D model showed a level of accuracy that was comparable but less than that shown by the two 3-D models.

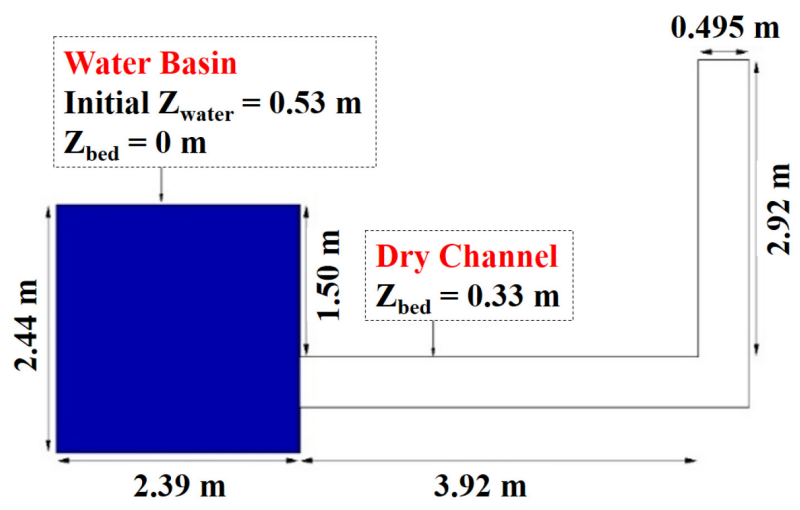

Fig. 1. Experimental setup and initial conditions for the dam break flow developing in a $90^{\circ}$ bend over a non-erodible flat channel bed. 

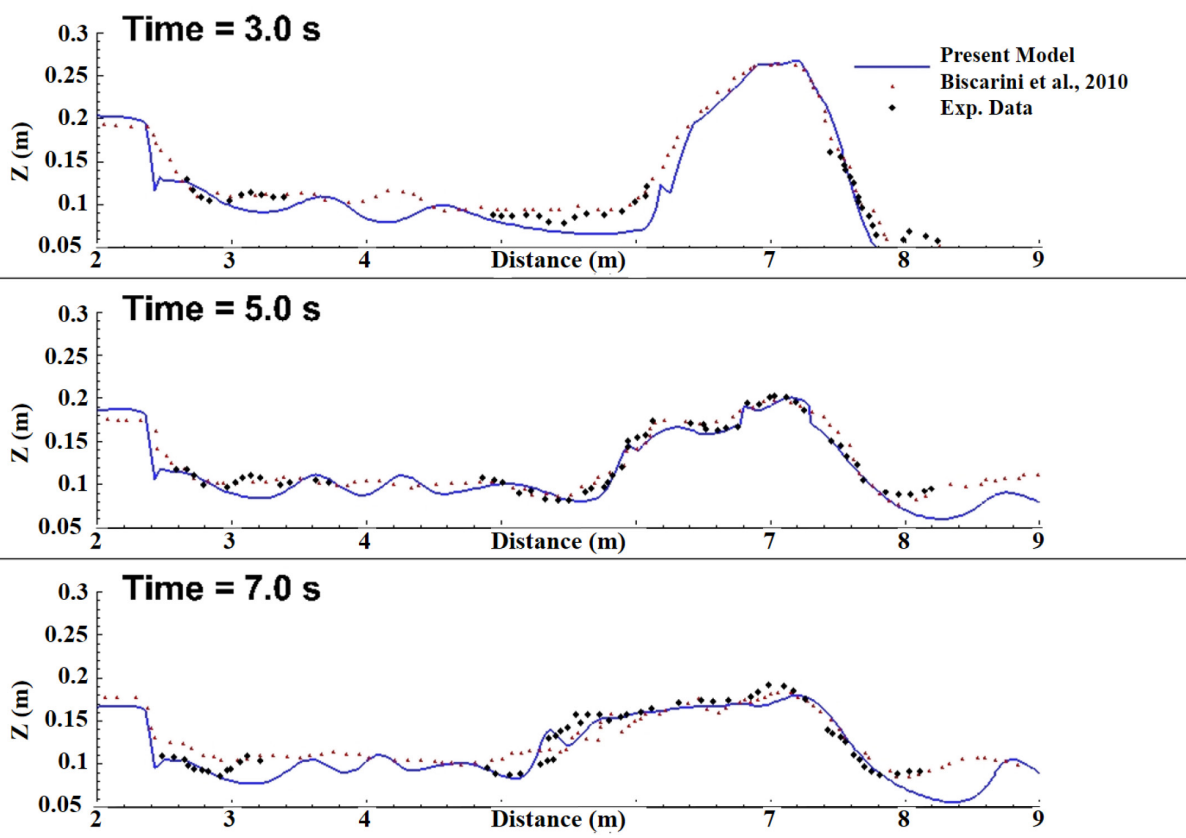

Fig. 2. Free-surface profiles predicted by the present 3-D model, the 3-D model proposed by Biscarini et al. (2010) and experimentally measured profiles at three different times after the start of the dam break flow developing in a $90^{\circ}$ bend.

A major goal of developing the present 3-D model is to be used as a design tool in evaluating the effectiveness of different flood-protection strategies. Many of these flood protection strategies are based on placing hydraulic structures like floodwalls or replacing existing structures (e.g., groynes, small hydraulic dams). Given that the presence of such hydraulic structures generally induces strong flow separation and increases the complexity of the flow, the use of a 3-D model is of high interest and should result in more accurate predictions. As an example, the flow in a domain containing a 7-km reach of the Iowa River near Iowa City was first simulated under a steady high flowrate $\left(\mathrm{Q}=1150 \mathrm{~m}^{3} / \mathrm{s}\right)$. Then, an unsteady sinusoidal hydrograph was propagated through the domain corresponding to a large flood with the peak discharge equal to the one of the 2008 flood in Iowa City. The computational mesh contained about 7 million cells. The mesh resolution in the horizontal direction was $5 \mathrm{~m}$ in the main channel of the Iowa River and $20 \mathrm{~m}$ in the floodplain, while maintaining a minimum of 20 cells in the vertical direction at initial conditions. Mesh refinement near solid boundaries was equal to 100 wall units. The specified time step was $0.1 \mathrm{~s}$. The 3-D model simulation was run with 64 processors for approximately 10 days. Here we present results for two simulations. In the first simulation (Figure 3 ) no floodwalls were present (Base Case). For the Base Case, aerial photographs of the flood extent observed in Iowa City for the same corresponding flowrate were taken during the 2008 flood. The flood extent was digitized and is plotted as a black line in Figure 3. Figure 3 also shows results obtained with a calibrated 2-D model for the same flowrate. Both the 3-D model as well as the calibrated 2-D model are able to reproduce with similar level of agreement the flood extent observed in the field. Errors in term of flooded area are of the order of $5 \%$ for both models.

In the second 3-D simulation (Figure 4), flood protection walls were placed in different areas over the floodplain where high economic developments are located (hospitals, university buildings). One can see that for the imposed hydrograph, the floodwalls do not allow flooding of the areas that need to be protected and, equally important, do not 
significantly increase flooding in other (non-protected) areas. The numerical models predicted that the floodwalls reduced by close to $25 \%$ the inundated area in the region of interest during the whole unsteady event.
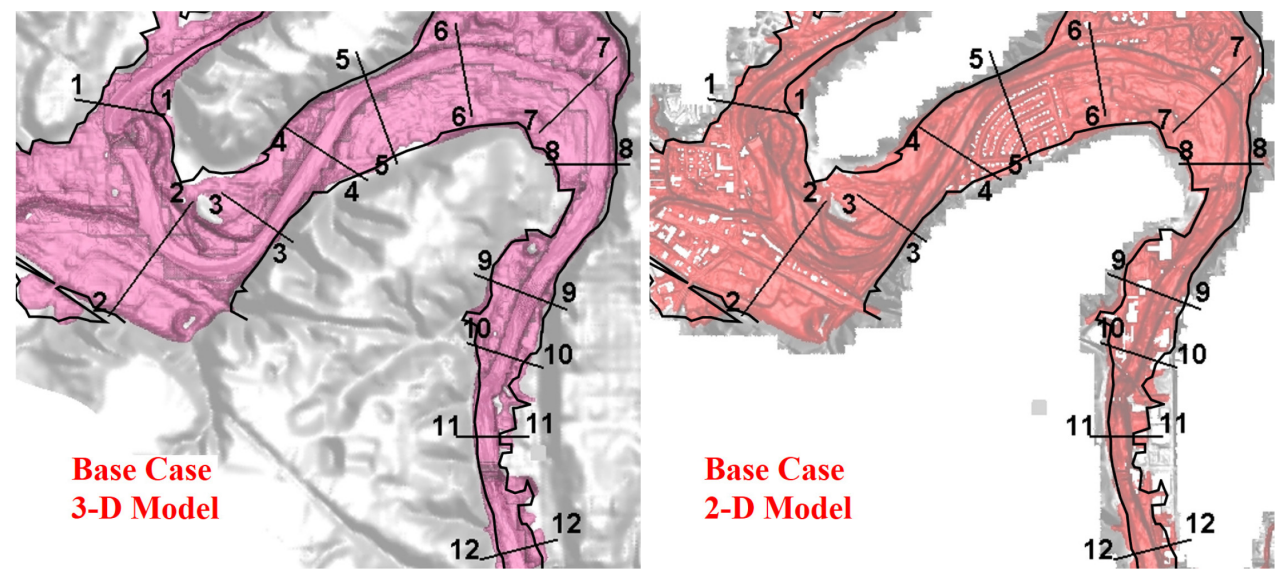

Fig. 3. Aerial view of flood extent in a domain containing a 7-km long reach of the Iowa River without flood protection walls at peak flood extent. Left: 3-D model solution. Right: 2-D model solution. Black lines represent flood extent for the same corresponding flowrate observed in Iowa City during the 2008 flood.

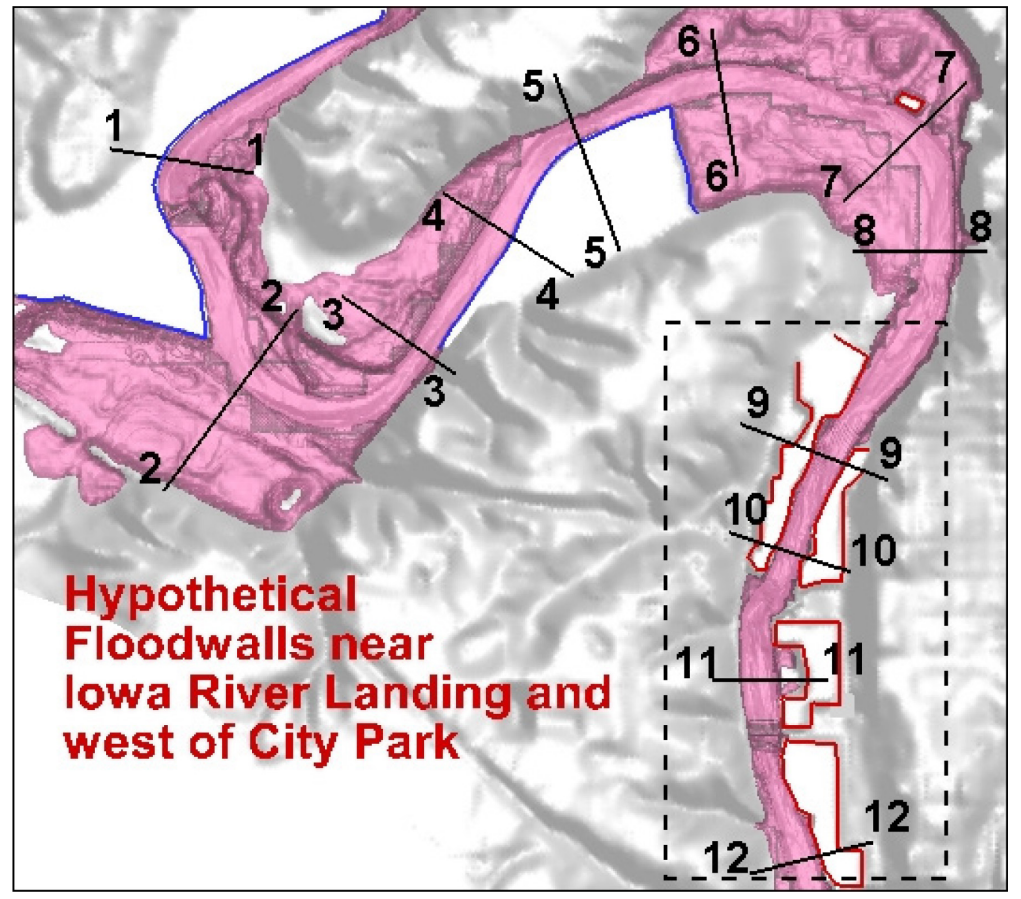

Fig. 4. Aerial view of flood extent in a domain containing a 7-km long reach of the Iowa River with flood protection walls (red and blue lines) at peak flood extent. 


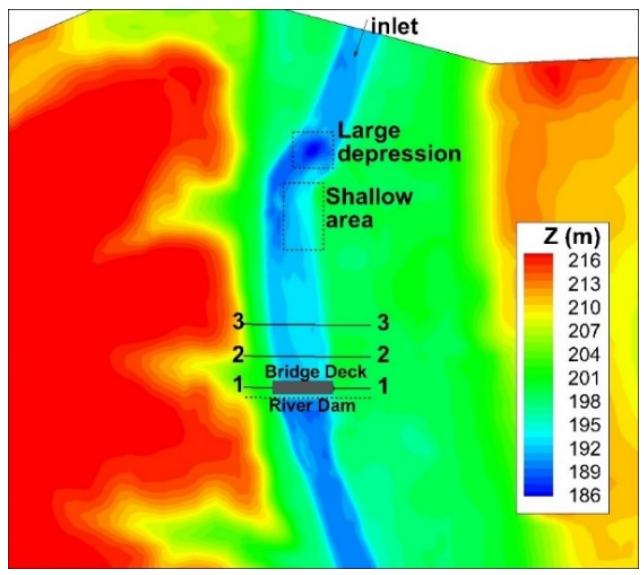

Fig. 5. Aerial view of the computational domain containing a $2 \mathrm{~km}$ long reach of the Iowa River and a bridge whose deck becomes submerged during the flooding event.

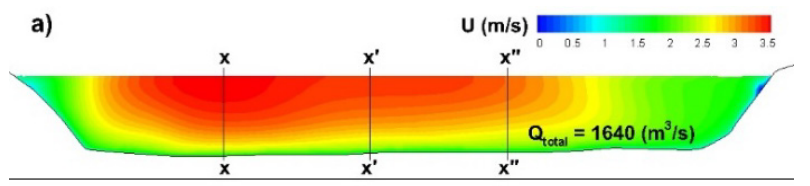

b)

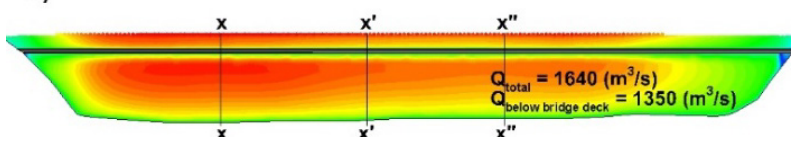

Fig. 6. Streamwise velocity distribution at Section 1 (middle of bridge deck, see Fig. 5): a) simulation without the bridge deck; b) simulation with the bridge deck showing results at peak discharge when the deck becomes submerged.

The next application considers a case in which the flow regime changes from open channel flow to pressurized flow because of the presence of a bridge deck that obstructs the passage of the flood wave. Occurrence of pressurized flow over an erodible bed is known to generate larger bed shear stresses and thus severe scour. These effects are referred to as pressure scour effects. While all previous test cases can also be calculated using 2-D models and such models can provide fairly accurate predictions of the main quantities of engineering interest, this is not the case when the flow becomes pressurized even locally around a hydraulic structure or a flow obstruction. In fact, 2-D models cannot be applied for such cases as all the main modelling assumptions are violated. The only way to obtain meaningful information on flow hydrodynamics and the capacity of the flow to entrain sediment is via a full 3-D simulation. In the test case reported in this paper, a bridge deck was placed in a 2$\mathrm{km}$ reach of the Iowa River near Iowa City (Figure 5) and a sinusoidal flood wave was propagated through this computational domain. The mesh resolution was consistent with the one explained for the previous case, with $5 \mathrm{~m}$ and $20 \mathrm{~m}$ resolution in the horizontal plane in the main channel and floodplain respectively while still maintaining at least 20 cells in the vertical direction at initial conditions. A total of about 3 million cells were used to mesh the computational domain. The additional consideration was to refine the cells near the boundary of the bridge deck, which was equivalent to 100 wall units. The time step was $0.1 \mathrm{~s}$ and the 3 -D model was run for approximately 3 days. To better understand the flow modifications induced by the bridge deck once it becomes submerged, an additional simulation in which the bridge deck was not present was performed with identical boundary conditions for the flood wave. During the time when the bridge deck became submerged, the velocity 
distribution changed dramatically with respect to the typical log-law profile that provides a fairly good approximation for the streamwise velocity distribution at times the bridge deck is not submerged (Figure 6). In the simulation with the bridge deck, once the deck becomes submerged the core of high velocities becomes wider and is situated closer to the bed. This explains why the erosive capacity of the flow during the time the deck is submerged in the simulation containing the bridge deck is about $80 \%$ higher than during the same time interval in the simulation containing no bridge deck.
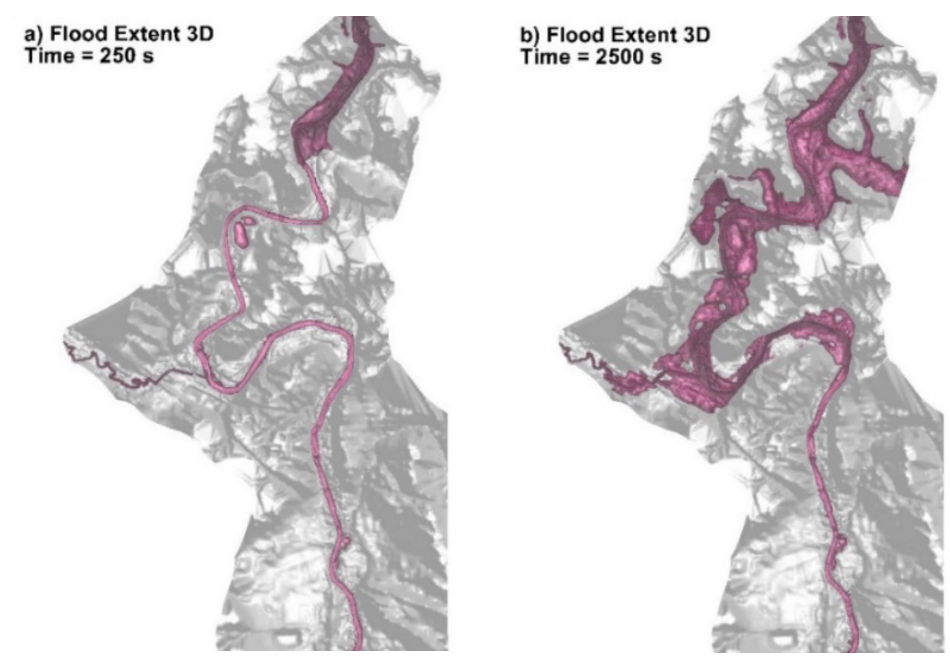

Fig. 7. Aerial view of flood inundation extent in a domain containing an 18-km long reach of the Iowa River near Iowa City caused by the collapse of the Coralville Dam. The flooded area is shown at two different times after the collapse of the dam: a) $250 \mathrm{~s}$; b) $2500 \mathrm{~s}$.

The final test case illustrates the use of the model to predict a realistic dam break scenario for the Coralville Dam, a flood-protection dam located upstream of the city of Iowa City, Iowa, USA. The elevation of the lake upstream of the Coralville Dam was assumed to be the maximum elevation ever recorded in the history of the dam. An instantaneous dam collapse was assumed. The dam break flow was allowed to propagate freely into the Iowa City area (approximately $18 \mathrm{~km}$ long). The mesh resolution varied across the domain. The horitzontal mesh resolution was of $100 \mathrm{~m}, 5 \mathrm{~m}$ and $20 \mathrm{~m}$ in the Coralville Lake, main channel of the Iowa River and floodplain respectively. A minimum of 40 and 20 cells in the vertical resolution were maintained in the Coralville Lake and Iowa River environment respectively at initial conditions. The total number fo cells was close to 18 million. The time step was $0.01 \mathrm{~s}$. The 3-D model was run with 2500 processors for a total of 1.5 months, in order to advance the solution to a physical time of $5 \mathrm{hrs}$. Aerial view snapshots of the dam break wave propagation toward Iowa City (the Coralville lake is not shown but it was part of the computational domain) are shown in Figure 7. One can also see that the flood wave moves upstream in the tributary joining the Iowa River once the front reaches the junction.

\section{Conclusions}

A fully 3D RANS non-hydrostatic model with deformable free-surface capabilities was developed within an existing CFD code to simulate flood propagation in natural river environments. This is one of the first applications of time accurate RANS for this type of problems. The paper reported validation of the model followed by its applications for several important classes of applications in river engineering and hydrology (flood propagation in river reaches and over their floodplains, investigation of pressure scour effects when the flow 
becomes pressurized over part of the domain, floods induced by a dam break). The 3-D model can also be used as an engineering tool to aid in deciding on the most effective design to implement a flood protection measure that may be under consideration by the authorities in charge of protecting against flood hazard and flood mitigation. Development of nested and hybrid modelling approaches integrating 1-D, 2-D and 3-D modelling approaches in various parts of the computational domain should be a main direction for future research in flood modelling and in particular in problems including failure of hydraulic structures that generate a flood wave whose evolution (e.g., dam break, levee breach), at least during its initial stages is subject to strong 3-D effects. Development of strategies to recalibrate 2-D and 1-D models based on 3-D simulations should also result in more accurate predictions using the lower order models.

\section{References}

1. C., Biscarini, S., Di Francesco, and P. Manciola. Hydrology and Earth System Sciences, 14, 705-708 (2010)

2. S., Soarez-Frazao, and Y. Zech, Journal of Hydraulic Engineering, 128(11), 956-968 (2002).

3. D., Horna-Munoz, D. and G., Constantinescu. A critical assessment of the performance of standard 2D flood models based on results of 3D URANS simulations, International Conference on Fluvial Hydraulics, River Flow 2016, Saint Louis USA (2016). 